\title{
Differential expression of parental alleles of BRCA1 in human preimplantation embryos
}

\author{
Pinar Tulay ${ }^{\star 1,2,4}$, Alpesh Doshi ${ }^{3}$, Paul Serhal ${ }^{3}$ and Sioban B SenGupta ${ }^{1,2}$
}

Gene expression from both parental genomes is required for completion of embryogenesis. Differential methylation of each parental genome has been observed in mouse and human preimplantation embryos. It is possible that these differences in methylation affect the level of gene transcripts from each parental genome in early developing embryos. The aim of this study was to investigate if there is a parent-specific pattern of $B R C A 1$ expression in human embryos and to examine if this affects embryo development when the embryo carries a BRCA1 or BRCA2 pathogenic mutation. Differential parental expression of $A C T B, S N R P N, H 19$ and BRCA1 was semi-quantitatively analysed by minisequencing in 95 human preimplantation embryos obtained from 15 couples undergoing preimplantation genetic diagnosis. BRCA1 was shown to be differentially expressed favouring the paternal transcript in early developing embryos. Methylation-specific PCR showed a variable methylation profile of $B R C A 1$ promoter region at different stages of embryonic development. Embryos carrying paternally inherited BRCA1 or 2 pathogenic variants were shown to develop more slowly compared with the embryos with maternally inherited BRCA1 or 2 pathogenic mutations. This study suggests that differential demethylation of the parental genomes can influence the early development of preimplantation embryos. Expression of maternal and paternal genes is required for the completion of embryogenesis.

European Journal of Human Genetics (2017) 25, 37-42; doi:10.1038/ejhg.2016.121; published online 28 September 2016

\section{INTRODUCTION}

Preimplantation embryo development follows a series of critical events, deprogramming of the genomes of sperm and egg and remarkable reprogramming of gene expression occurs to activate the embryonic genome. With the exception of imprinted loci, the expression of both maternal and paternal copies of genes is required for the completion of embryogenesis. ${ }^{1}$ In mice, the genome of the oocyte is markedly undermethylated compared with the sperm. ${ }^{2}$ Upon fertilization, mammalian zygotes (including humans) undergo genome-wide demethylation to establish the pluripotency of the newly developing embryo. ${ }^{2}$ Selective demethylation of the male pronucleus occurs upon fertilization. ${ }^{3-9}$ In contrast to the male pronucleus, demethylation of the female mouse pronucleus starts with the first cleavage divisions. ${ }^{2,3,7-10}$ Recent genome-wide DNA methylation studies have reported a wave of demethylation in early preimplantation embryos. Throughout early embryonic development, a differential methylation pattern is maintained in the majority of the differentially methylated regions in imprinted genes, although some show stagespecific changes. ${ }^{11}$ Repetitive elements, housekeeping genes and genes controlling pluripotency or differentiation have been reported to have specific methylation patterns during embryo development. ${ }^{12,13}$

We hypothesize that, during the transition of demethylation and deprogramming in preimplantation embryo development, there is differential expression of parental alleles in certain genes that are not imprinted genes. The differential expression of parental alleles arises due to variation in the timing of demethylation and the level of methylation of each parental genome. Changes in the methylation patterns of BRCA1 have been reported in early developing preimplantation embryos. ${ }^{14}$ If there is differential demethylation and remethylation of non-imprinted genes of maternal and paternal genomes during early development, then the level of transcription from each parental genome may also be different. Thus, when a pathogenic mutation is present, the differential level of mutant and normal transcripts available for translation in the early embryo will be determined by the parental origin of the variant in the embryo. This, in turn, suggests that the effect of inheritance of a variant may vary in the early embryo, depending on whether it was transmitted from the maternal or paternal genome.

We, therefore, sought to characterize differential parental gene expression in human preimplantation embryos obtained from patients undergoing preimplantation genetic diagnosis (PGD) and to investigate the possible effect of a pathogenic variant on embryo development depending on the parental inheritance of the variant.

\section{MATERIALS AND METHODS}

Sample collection and processing

This study was licensed by the Human Fertilization and Embryology Authority (Reference: RO113) and ethical approval was granted by the National Research Ethics Service, Research Ethics Committee (Reference: 10/H0709/26). Wholeblood and surplus embryos were collected from couples who had given informed consent following PGD treatments for a variety of monogenic disorders.

Ovarian reserve tests and gonadotrophin stimulation were performed as described previously. ${ }^{15}$ Briefly, immature oocytes were matured in G-IVF Plus medium (Vitrolife, Göteborg, Sweden) within $4 \mathrm{~h}$ of collection. Intracytoplasmic sperm injection was performed $\sim 40 \mathrm{~h}$ post hCGH injection. Fertilization was assessed at $16-20 \mathrm{~h}$ post insemination and the presence of two pronuclei and

\footnotetext{
${ }^{1}$ Near East University, Faculty of Medicine, Department of Medical Genetics, Yakin Dogu Bulvari, Nicosia, Cyprus; ${ }^{2}$ UCL Preimplantation Genetics Group, Institute for Women's Health, University College London, London, UK; ${ }^{3}$ The Centre for Reproductive and Genetic Health, The New Wing Eastman Dental Hospital, London, UK

${ }^{*}$ Correspondence: Dr P Tulay, Near East University, Faculty of Medicine, Department of Medical Genetics, Yakin Dogu Bulvari, Nicosia, Cyprus.

Tel: +357 22 00903924440535; Fax: +357 22 00903922236461; E-mail: pinar.tulay@ucl.ac.uk

${ }^{4}$ Current address: Near East University, Faculty of Medicine, Department of Medical Genetics, Yakin Dogu Bulvari, Nicosia, Cyprus.

Received 2 October 2015; revised 1 July 2016; accepted 5 August 2016; published online 28 September 2016
} 
polar bodies indicated normally fertilized oocytes. ${ }^{16}$ Embryos were cultured in G-1/G-2 PLUS media (Vitrolife). Preimplantation embryos were graded according to Bolton et al. ${ }^{17}$ Those embryos diagnosed as affected following PGD or not suitable for transfer were collected on day 6 post fertilization, in order to be used for this project. Each embryo was washed and transferred in phosphate buffered saline with $0.1 \%$ polyvinyl alcohol solution (PBS/PVA, Sigma, St Louis, MO, USA) and $0.3 \mathrm{U} / \mu \mathrm{l}$ RNasin plus RNAse inhibitor (Promega, Southampton, UK).

\section{Genotyping couples}

DNA extracted from whole blood from the couple was sequenced (BigDye Terminator v3.1 Cycle Sequencing, ABI, Barnsley, UK) for exonic regions of GAPDH, ACTB, UBE3A, SNRPN, IGF2, H19 and BRCA1 to identify informative single-nucleotide polymorphisms (SNPs) between the partners (Supplementary Table 1). A couple was defined as fully informative for an SNP when each partner was homozygous for different alleles, whereas a couple was defined as semi-informative when one partner was homozygous and the other was heterozygous with one shared allele at the SNP. The data were submitted to the gene variant database at www.LOVD.nl/CAV3 (Patient IDs-variant IDs: 00074438-0000119134, 00074441-0000119305, 00074442-0000119307, 000744430000119309, 00074444-0000119310 and 00074445-0000119312).

\section{DNA and RNA extraction from embryos}

DNA and RNA from embryos were extracted using the AllPrep DNA/RNA micro kit (Qiagen, Manchester, UK). The quality of RNA was assessed using the RNA 6000 Pico kit (Agilent, Oxford, UK) on a Eukaryote Total RNA Pico Series II chip using the Agilent 2100 Bioanalyzer (Agilent, Colorado Springs, CA, USA).

\section{Analysis of differential gene expression}

Reverse transcription of RNA obtained from the embryos was performed using the SuperScript III first-strand synthesis system for RT-PCR (Invitrogen, Paisley, UK). DNA contamination was identified by multiplex PCR (Qiagen) using two markers (Eurogentec, Camberley, UK) D19S112 and APOC2. These markers were selected to detect DNA contamination as they amplify an exonic as well as an intronic region. In addition, these markers were used due to the high heterozygosity.

\section{SNaPshot minisequencing assay sensitivity}

Differential expression of parental transcripts using RNA samples was analysed semi-quantitatively on the previously identified informative and semiinformative SNPs by SNaPshot minisequencing assay (Applied Biosystems, Warrington, UK) (Supplementary Table 2). Differential expression of one parental transcript relative to the other was defined as an allele peak height ratio $>1: 2$. Monoallelic or preferential allelic expression of a transcript was only considered in embryos where both parental alleles could be identified in the analysed SNP. If an allele shared by each parent was expressed in the embryo, the sample was excluded from the analysis. Statistical analysis was performed by Student's $t$-test using GraphPad prism v6 software (San Diego, CA, USA). The quantitative difference between each parental allele in the embryos identified by minisequencing was validated by real-time PCR with subsequent highresolution melting analysis (Roche, Welwyn Garden City, UK).

\section{Chromosomal copy number analysis}

The chromosome copy number of the genes (chromosomes 7, 11, 15 and 17) was analysed using DNA to ensure that the differential expression detected is not due to an aneuploidy in the embryo. The copy number of chromosomes 7 , 11,15 and 17 was determined by haplotype analysis. Polymorphic markers that are on the same chromosome with the genes analysed, ACTB, SNRPN, H19 and $B R C A 1$, and that were available in our laboratory were used to determine the chromosome copy number. Analysis of the copy number of chromosome 7 was linked to CFTR (D7S2420, D7S2459 and D7S486), chromosome 11 markers were linked to HBB (D11S1338, D11S1997 and D11S4147), chromosome 15 to FBN1 (D15S992, D15S123 and D15S94) and chromosome 17 to BRCA1 (D17S579, D17S1789, D17S1353 and D17S841). The copy number of the chromosomes was scored only if the origin of the parental alleles at that locus could be distinguished. In these cases, if only one parental allele was detected, the embryo was considered to have lost the copy of the chromosome harbouring the missing allele. Embryos were considered to have gained a chromosome when three alleles were detected. An isodisomy of the chromosome could not be detected in the embryo using this method.

A subset of embryos was also analysed by array comparative genomic hybridization $(\mathrm{aCGH})$ using the 24Sure system, following whole-genome amplification (BlueGnome, Cambridge, UK). The slides were scanned using ScanArray Express (Perkin Elmer, Danbury, CT, USA) and the arrays were analysed using Bluefuse Multi analysis software v.2.6 (BlueGnome). The cutoff of the $\log 2$ ratio fluorescent test signal over the control DNA was set as +0.3 for the gain and -0.3 for the loss of a chromosome by the software.

\section{Methylation studies}

The methylation status of $A C T B, H 19$ and BRCA1 was analysed in a subset of embryos. The EpiTect Bisulfite conversion kit (Qiagen) was used for DNA treatment. A set of outer primers with no $\mathrm{CpG}$ dinucleotides was designed for $A C T B, H 19$ and BRCA1 (Supplementary Table 3). Two sets of inner primers resulting in PCR products of different sizes directed to the methylated and unmethylated sequences were used for the promoter regions of $A C T B, H 19$ and BRCA1. $^{18}$

\section{Statistical analyses: embryos with BRCA1 or 2 pathogenic} mutations and preimplantation embryo development

The developmental stage of all the embryos with BRCA1 or 2 pathogenic mutations was examined on day $5 / 6$ post fertilization and related to the parental inheritance of the variant. Statistical analysis was performed to investigate the difference between the developmental stage of embryos with paternally inherited $B R C A 1$ or 2 pathogenic mutations and the maternally inherited $B R C A 1$ or 2 pathogenic mutations by $\chi^{2}$-test using GraphPad prism v6 software.

\section{RESULTS}

\section{Genotyping analysis}

Parental genotyping by sequencing of seven genes identified informative SNPs between 15 partners that enabled detection of heterozygous embryos in four genes (Table 1).

Haplotyping analysis was performed to determine the copy number of chromosomes 7, 11, 15 and 17 in embryos. The detailed results are listed in Table 2. Of these embryos, 26 were also analysed by aCGH. Twelve embryos were shown to be euploid and the rest of the embryos showed various aneuploidies. Only two of these embryos (embryo 69 and 72) showed aneuploidies for the chromosomes of interest (gain of chromosome 17) and these embryos were excluded from the analysis (Table 2).

\section{Minisequencing assay sensitivity for allelic imbalance}

The sensitivity of the minisequencing analysis was validated by realtime PCR. Amplification of cDNA from the embryos showed that the mean Cq values for the start of the exponential phase of amplification were 36 for ACTB, 34 for SNRPN, 41 for H19 and 35 for BRCA1. Therefore, PCR prior to minisequencing analysis was stopped before the exponential phase was reached. High-resolution melting analysis of all the PCR products from embryos confirmed the allelic imbalances identified by minisequencing.

\section{Differential gene expression in preimplantation embryos}

A total of 95 embryos were analysed to establish the parental expression profiles of $A C T B, S N R P N, H 19$ and BRCA1. A summary of the results is shown in Table 1 and Figure 1. The expression level of maternal and paternal transcripts of ACTB was similar for all 11 embryos analysed for this gene.

Minisequencing analysis of imprinted gene transcripts showed that paternal transcript of SNRPN predominated in more than half of the embryos regardless of their developmental stage $\left({ }^{\star} P=0.01 ; 56.5 \%\right.$, 
Table 1 Summary of differential expression analysis

\% of embryos showing differential expression favouring (number of embryos)

\begin{tabular}{|c|c|c|c|c|c|}
\hline Genes analysed & $\begin{array}{l}\text { Number of embryos } \\
\text { included for the study }\end{array}$ & $\begin{array}{l}\% \text { of informative haplotypes } \\
\text { (number of embryos) }\end{array}$ & Paternal & Maternal & Similar \\
\hline ACTB & 30 & 36.7 (11) & 0 & 0 & $100(11)$ \\
\hline SNRPN & 34 & $67(23)$ & $56.5(13)^{a}$ & $17(4)$ & $26(6)$ \\
\hline H19 & 48 & $33(15)$ & $13(2)$ & $60(9)^{a}$ & $26(4)$ \\
\hline$B R C A 1$ & 75 & $64(49)$ & $58(29)$ & $19(9)$ & $22(11)$ \\
\hline
\end{tabular}

anly the paternal expression of SNRPN was observed in 9/13 embryos and only the maternal expression of $H 19$ was observed in 7/9 embryos. The genes analysed for differential expression, percentage of informative haplotypes where each parental allele was distinguished and the percentage of embryos with differential gene expression favouring the paternal and maternal transcripts are listed. BRCA1 was analysed at two loci and the results shown here were collective from both loci. In 10 embryos, differential expression of BRCA1 at two loci did not agree and these were not included in the analysis.

Table 2 Table summarizing the embryos analysed for haplotyping analysis for the chromosomes of 7 (CFTR), 11 (HBB), 15 (FBN1) and 17 (BRCA1)

\begin{tabular}{|c|c|c|c|c|}
\hline $\begin{array}{l}\text { Chromosomes } \\
\text { analysed }\end{array}$ & $\begin{array}{l}\text { Number of embryos } \\
\text { included for the study }\end{array}$ & $\begin{array}{l}\% \text { of informative haplotypes } \\
\text { (number of embryos) }\end{array}$ & $\begin{array}{l}\% \text { of heterozygous embryos } \\
\text { (number of embryos) }\end{array}$ & $\begin{array}{c}\% \text { of embryos with amplification failure } \\
\text { (number of embryos) }\end{array}$ \\
\hline 7 & 30 & $86.7(26)$ & 86.7 (26) & $13(4)$ \\
\hline 11 & 34 & $53(18)$ & 94 (17) & $26(9)$ \\
\hline 15 & 48 & $25(12)$ & 91 (11) & $4(2)$ \\
\hline 17 & 75 & $68(51)$ & $94(48)$ & $19(21)$ \\
\hline
\end{tabular}

Number of embryos included in the haplotyping analysis with the percentage of informative haplotypes and heterozygote embryos are summarized. The percentage of inconclusive analysis due to amplification failure was also shown.
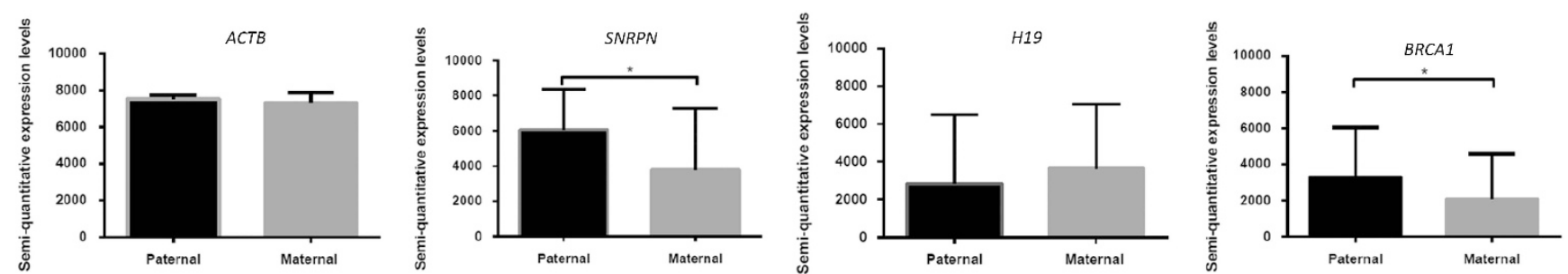

Figure 1 Overall differential expression of paternal and maternal transcripts for four genes in human embryos. It was shown that there was no significance in the differential expression of parental transcripts for ACTB $(P=0.2)$. Paternal SNRPN transcripts were expressed at significantly higher levels relative to the maternal transcripts $\left({ }^{*} P=0.01\right)$ in embryos. Although higher levels of maternal $H 19$ transcripts were detected in embryos, this was not significant $(P=0.4)$. The differential expression of paternal BRCA1 expression was shown to be significant relative to the maternal transcripts $\left({ }^{*} P=0.03\right)$ in the embryos.

13/23, Figures 1 and 2). Monoallelic expression of paternal SNRPN transcript was observed in $69 \%(9 / 13)$ of these embryos. As the embryos reached later stages of development, differential expression favouring the paternal SNRPN transcript increased from $61 \%(8 / 13)$ at cleavage and morula stages to $70 \%(7 / 10)$ for the blastocyst stage embryos.

Unlike SNRPN, H19 was not readily detected in human preimplantation embryos $(31 \%, 15 / 48)$. One embryo was excluded from the analysis as it was shown to have only the maternal copy of chromosome 11 by haplotyping. This accounted for the detection of only the maternal $\mathrm{H} 19$ transcript by the SNapShot assay. Overall, $60 \%$ (9/14) of the embryos expressed predominantly the maternal H19 transcript with $78 \%$ (7/9) being strictly monoallelic for the maternal transcript. Preferential expression of the maternal $H 19$ transcript was observed to be $50 \%(2 / 4)$ at cleavage, $66 \%$ (4/6) in morula and $75 \%$ (3/4) in blastocyst stage embryos.

Two SNPs, located in exon 11 and exon 12, were analysed to investigate differential expression of parental BRCA1 transcripts. About $13 \%(10 / 75)$ of the embryos analysed were excluded from the analysis as the differential expression was not concordant at these two SNPs. Two embryos were excluded from the analysis as they showed a gain of chromosome 17, reflecting the preferential BRCA1 expression by the SNaPshot assay. Differential BRCA1 expression in the embryos was $66 \%(10 / 15)$ at cleavage, $55 \%(10 / 18)$ at morula and $50 \%(7 / 14)$ at the blastocyst stage. Overall, there were significantly more embryos with elevated expression of paternal BRCA1 transcripts compared with embryos with increased expression of maternal transcripts, regardless of the developmental stage $\left({ }^{\star} P=0.03\right.$, Figures 1 and 2).

\section{Differential methylation analysis}

Methylation analysis was performed by bisulfite conversion, followed by methylation-specific PCR. Due to insufficient starting material, the amount of DNA obtained from a single embryo and the bisulfite treatment being deleterious for DNA, sequencing analysis on the converted DNA could not be performed. The promoter regions of three genes, ACTB, H19 and BRCA1, were amplified by nested PCR. When monoallelic expression of one parental transcript was observed 
a

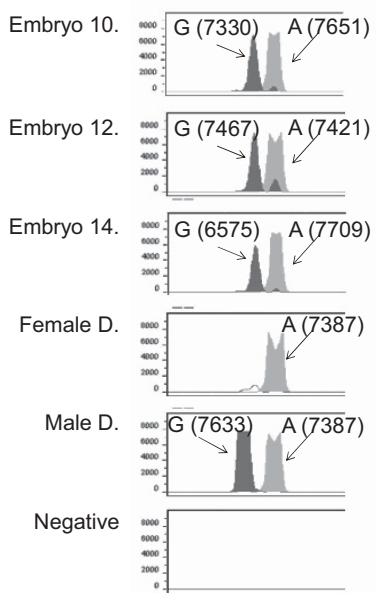

b

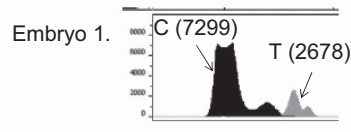

Female A.

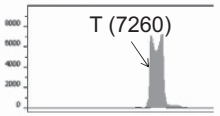

Male A.

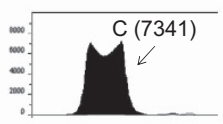

Negative

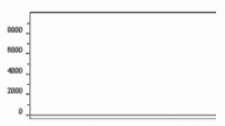

C

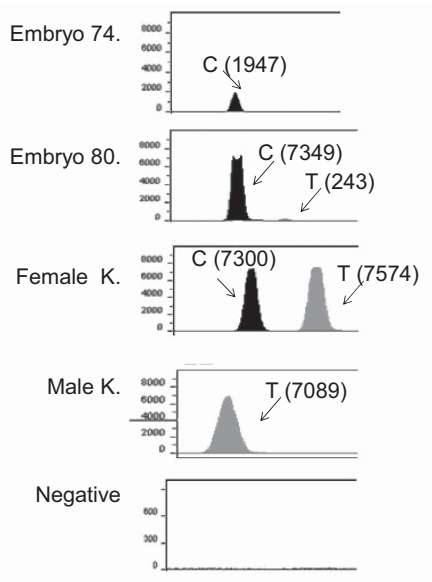

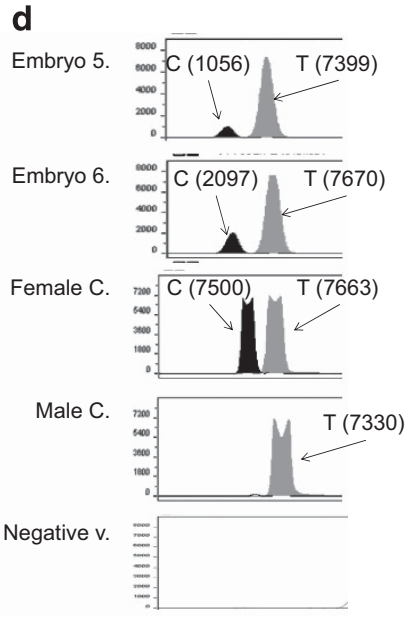

Figure 2 GeneScan (Cheshire, UK) fragment size analysis result panels showing differential gene expression of: (a) ACTB from couple D. The panel shows the embryo number 10, 12 and 14 (all heterozygote for $G$ and A) with similar expression levels of the parental copies of the SNP analysed, female partner (homozygote for A), male partner (heterozygote $G$ and $A$ ) of couple $A$ and negative control with no DNA. (b) SNRPN from couple A. The panel shows the embryo number 1 (heterozygote for $G$ and A) with increased expression of the paternal copy of the SNP analysed, female partner (heterozygote for $G$ and A), male partner (homozygote for G) of couple A and negative control with no DNA. (c) H19 from couple K. The panel shows the embryo number 74 (maternally expressed allele only, homozygote for $\mathrm{C}$ ) and 80 (heterozygote for $\mathrm{C}$ and $\mathrm{T}$ ) with increased expression of the maternal copy of the SNP analysed, female partner (heterozygote for $\mathrm{C}$ and $\mathrm{T}$ ), male partner (homozygote for $\mathrm{T}$ ) and negative control with no DNA. (d) BRCA1 from couple $\mathrm{C}$. The panel shows the embryo number 5 and 6 (both heterozygote for $C$ and T) with considerably increased expression of the paternal copy of the SNP analysed, female partner (heterozygote for $\mathrm{C}$ and $\mathrm{T}$ ), male partner (homozygote for $\mathrm{T}$ ) and negative control with no DNA. The panels show the alleles expressed in the embryo and the allele of the SNP of the female and male partner of the couple. The peak heights of the alleles are shown in parentheses.

by $\mathrm{SNaPshot}$ analysis, a hemi-methylated profile was expected to be observed as only one parental transcript is present. When both parental transcripts were expressed at similar levels by SNaPshot analysis, an unmethylated profile was expected to be observed, representing both parental transcripts at similar levels. As allelespecific methylation was not studied, it is not possible to draw a definite conclusion as to whether the methylation status was the reason for differential gene expression.

Methylation analysis of $A C T B$ in five embryos showed that these embryos were unmethylated, which supports the expression profile observed by SNaPshot analysis (Figure 3). Methylation analysis of 3/8 embryos confirmed the differential H19 expression. Of these embryos, two were hemi-methylated. These embryos also showed monoallelic expression of $H 19$ transcript. One embryo was unmethylated, and this supported the biallelic expression of $\mathrm{H} 19$ transcripts by the minisequencing assay. In 5/8 embryos, differential expression of the parental transcripts did not relate to the methylation status. The hemi-methylated profile of BRCA1 confirmed differential expression of parental transcripts in 58\% (18/31) of the embryos.

\section{Development of embryos with $B R C A 1$ and $B R C A 2$ variants}

Following PGD for BRCA1 and BRCA2 in six couples, 31 embryos were identified with a $B R C A 1$ or $B R C A 2$ pathogenic mutations. Half of the embryos with paternally transmitted BRCA1 germline pathogenic mutations (8/16) were arrested at the cleavage stage. Only $12.5 \%$ $(2 / 16)$ of these embryos reached the blastocyst stage 5 days post fertilization. Embryos with maternally inherited BRCA1 variants developed at a significantly faster rate $(8 / 15,53 \%$ at blastocyst stage, $P=0.01)$ compared with the embryos with paternally inherited $B R C A 1$ and BRCA2 pathogenic mutations (Figure 4).

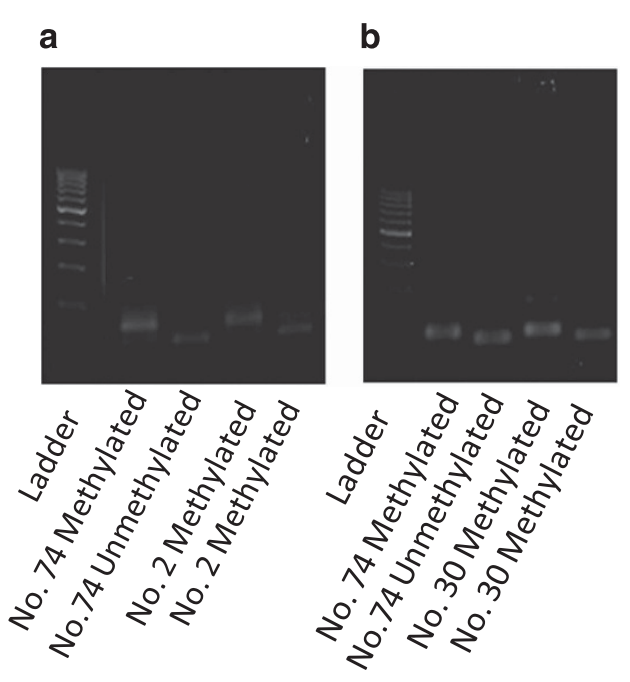

Figure 3 Agarose gel electrophoresis of embryos showing partial methylation for (a) H19 and (b) BRCA1. Lanes 1 for images (a) and (b) represent 100 base pair ladder and the rest of the lanes represent the methylation PCR product results of DNA obtained from embryos following bisulfite conversion. Embryo numbers are labelled for each lane and the PCR directed towards the methylated DNA is represented as 'methylated' and the unmethylated DNA as 'unmethylated'.

\section{DISCUSSION}

Differential expression

This study showed that similar levels of parental ACTB transcripts were expressed in human embryos. The paternally imprinted gene H19 was not always detected in embryos. Some studies have reported the detection of both parental alleles in slow-growing embryos or 


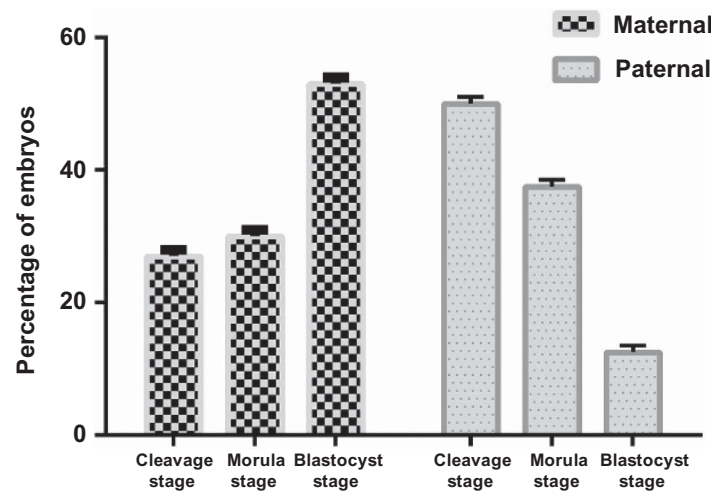

Figure 4 Developmental rate of embryos carrying paternally inherited $B R C A 1$ or 2 pathogenic mutations compared with maternally inherited BRCA1 or 2 pathogenic mutations. Significantly fewer embryos developed to the later stages of preimplantation development (morula and blastocyst stages) compared with the embryos carrying maternally inherited mutations $\left({ }^{*} P=0.01\right)$.

morphologically poor embryos, ${ }^{19}$ whereas some did not detect $H 19$ at all. ${ }^{20}$ In this study, $H 19$ was detected in $31 \%(15 / 48)$ of the embryos. Although biallelic expression of the H19 transcript (7/14) was detected in half of the embryos confirming previously published reports ${ }^{21}$ preferential expression of maternal $H 19$ transcripts was observed in the majority of the embryos. Although it is well accepted that $H 19$ is a paternally imprinted gene, expression of both parental $\mathrm{H} 19$ alleles has been reported in the human oocytes and preimplantation embryos. Studies have suggested that developmentally delayed embryos show an unexpected expression and methylation profile. ${ }^{19}$ In this study, embryos with similar parental expression of $H 19$ developed at a slower rate where seven embryos were between 5 and 10 cell stage and two were at morula stage on day $5 / 6$ post fertilization. In addition, all of the embryos that reached blastocyst stage showed opposing patterns of parental expression for these two imprinted genes. This observation supports the finding of Khoueiry et $a l^{21}$ who reported that slow-developing embryos had a balanced pattern of methylated and unmethylated strands of H19DMR.

The imprinting of SNRPN was not completed in the early developing preimplantation embryos, such that the maternal SNRPN transcript was detected in $60 \%(9 / 15)$ of the embryos, as reported previously. ${ }^{20,22}$ The unexpected expression of maternal SNRPN alleles could be due to the on-going maternal mRNA degradation in these embryos, whereas the paternal H19 transcripts could be caused by partial resetting of $\mathrm{H} 19$ in the sperm. ${ }^{19}$ The onset of the monoallelic expression of these genes might be at a later stage in human embryos or the time of the monoallelic expression could be variable among embryos. It has been reported that the time of the monoallelic expression of the imprinted genes, such as IGF2, SNPRN and MEST, varies in mouse embryos. ${ }^{22,23}$ Developmentally delayed embryos were shown to have unusual expression and methylation profiles. ${ }^{19,21}$ This was also observed in our study, where $20 \%$ (3/15) of slow-developing embryos had similar expression levels of $H 19$ and 39\% (9/23) of slowdeveloping embryos had similar parental levels of SNRPN transcripts. None of these embryos reached the blastocyst stage. It is also possible that assisted reproductive technology techniques lower the level of methylation for the imprinted genes, ${ }^{19,24-26}$ causing the unexpected expression of transcripts. ${ }^{27}$

In this study, differential expression of paternal $B R C A 1$ transcript was observed in embryos. This preferential expression was more prevalent in the cleavage-stage embryos. As the embryos developed to the blastocyst stage, differential expression of $B R C A 1$ was reduced. In $13 \%$ of the embryos, differential BRCA1 expression could not be determined as the expression profiles for the two SNPs analysed were not concordant. BRCA1 is known to undergo alternative splicing in a number of its exons, forming isoforms that skip exon 5, exons 2-10, exons 9-11, exon 11 only, exons $14-17$ and exons 14-18. ${ }^{28,29}$ Alternative splicing of exon 11 yields a full-length isoform and also shorter isoforms either through the use of an alternative intra-exonic splice donor site en-31 $^{29}$ through complete skipping of exon 11 . Therefore, if one of these isoforms was present in the embryos analysed in this study, differences in the expression profiles of BRCA1 in exon 11 and exon 12 may have arisen.

The methylation analysis showed that preferential expression observed in H19 and BRCA1 may be due to the methylation status of the parental transcripts. A gradual demethylation was previously observed for BRCA1 during cleavage divisions of human embryos where $\sim 30 \%$ of the methylated BRCA1 residues remained up to the blastocyst stage. ${ }^{14}$ A recently published study also showed that monoallelic as well as biallelic expression was detected in human primary fibroblasts. ${ }^{32}$ They reported that each cell mostly expressed one allele. The abundance of the cellular transcripts and the monoallelic expression could account for phenotypic variability in humans including penetrance and expressivity of a dominant developmental disorder, or cellular heterogeneity in cancers and predisposition to a complex phenotype. ${ }^{32}$

\section{Development of embryos with $B R C A 1$ and $B R C A 2$ pathogenic mutations}

The development of the embryos with $B R C A 1$ or 2 pathogenic mutations were investigated in six couples undergoing PGD. Three males and three females with BRCA1 or 2 pathogenic mutations opted for PGD. Two of the male partners had BRCA1 pathogenic mutations and one had BRCA2 pathogenic mutations. The female partner of the rest of the couples undergoing PGD had BRCA1 pathogenic mutations. The majority of the embryos with paternally inherited $B R C A 1$ or 2 pathogenic mutations (eight embryos with BRCA1 and eight embryos with $B R C A 2$ pathogenic mutations, respectively) were shown to arrest at the cleavage between 4 and 10 cell stages (50\%). Only $38 \%$ of the embryos developed to the morula stage and only $12.5 \%$ of the embryos reached the blastocyst stage. Embryos with paternally $B R C A 1$ - and BRCA2-inherited pathogenic mutations (16 embryos) were shown to develop significantly slower compared with embryos with maternally inherited variants $\left(15\right.$ embryos, $\left.{ }^{\star} P=0.01\right)$. As the paternal genome undergoes a rapid demethylation starting at the early stages of preimplantation embryos, embryos with paternally inherited $B R C A 1$ and BRCA2 pathogenic mutations and defective homologous recombination pathways may be prevented from developing to the later stages of preimplantation development. However, embryos with maternally inherited BRCA1 pathogenic mutations may compensate for the variant and initiate homologous recombination repair through paternal transcripts that were free from the variant. Therefore, when the embryos are carrying a paternally inherited BRCA1 or BRCA2 pathogenic mutations, it may be more prone to embryonic lethality during cleavage divisions. Thus, we speculate that less viable embryos with paternally inherited BRCA1 or 2 pathogenic mutations are produced compared with the maternally inherited $B R C A 1$ or 2 pathogenic mutations. Therefore, we assume that there are more adults with maternally inherited $B R C A 1$ or 2 pathogenic mutations. The higher number of maternally inherited $B R C A 1$ or 2 variants was also reported previously. ${ }^{33,34}$ Although these articles were evaluating the risk of cancer and the onset of cancer depending on the parental 
origin of the $B R C A 1$ or 2 variants, their data showed that there were more patients with maternally inherited $B R C A 1$ or 2 variants. ${ }^{33,34}$ Once the genome-wide demethylation of genes is completed during cleavage divisions, we assume that embryos with maternally and paternally inherited $B R C A 1$ or 2 variants have similar chances of implantation and pregnancy.

\section{CONCLUSION AND FUTURE PERSPECTIVES}

The main limitation of this study was the small number of embryos analysed due to the scarcity of the human embryos. However, even with this small number of embryos, these data show significant outcome relating gene expression with development of preimplantation embryos. This study showed that SNRPN, H19 and BRCA1 transcripts were differentially expressed in human embryos. The presence of a $B R C A 1$ or 2 pathogenic mutations inherited from the paternal genome had a greater detrimental effect on the development of the embryo to blastocyst compared with pathogenic mutations inherited from the maternal genome. This may stem from differences in methylation patterns of the parental genomes in embryos. Therefore, the contribution of the paternal genome in the preimplantation embryo development may be vital, especially in the early stages. Further extrapolation of this data suggest that the risk of transmitting a $B R C A 1$ or 2 pathogenic variants may be altered by the parental origin of the variant. Paternally transmitted $B R C A 1$ or 2 pathogenic mutations are more likely to result in embryos that fail to reach blastocyst, thereby limiting the implantation potential of these embryos. Consequently this may lower the overall risk of males with BRCA1 or 2 mutations having children who have inherited their pathogenic mutation.

\section{CONFLICT OF INTEREST}

The authors declare no conflict of interest.

\section{AUTHOR CONTRIBUTIONS}

PT and SSB: substantial contributions to conception and design. PT, AD and PS: acquisition of data. PT and SSB: interpretation of data. PT: analysis and drafting the article. PT, AD, PS and SSB: revising it critically for important intellectual content, and final approval of the version to be published.

1 McGrath J, Solter D: Completion of mouse embryogenesis requires both the maternal and paternal genomes. Cell 1984; 37: 179-183.

2 Monk M, Boubelik M, Lehnert S: Temporal and regional changes in DNA methylation in the embryonic, extraembryonic and germ cell lineages during mouse embryo development. Development 1987; 99: 371-382.

3 Rougier N, Bourc'his D, Gomes D M et al: Chromosome methylation patterns during mammalian preimplantation development. Genes Dev 1998; 12: 2108-2113.

4 Dean W, Santos F, Stojkovic M et al: Conservation of methylation reprogramming in mammalian development: aberrant reprogramming in cloned embryos. Proc Natl Acad Sci USA 2001; 98: 13734-13738.

5 Swald J, Engemann S, Lane $\mathrm{N}$ et al: Active demethylation of the paternal genome in the mouse zygote. Curr Biol 2000; 10: 475-478.

6 Mayer W, Niveleau A, Walter J, Fundele R, Haaf T: Demethylation of the zygotic paternal genome. Nature 2000; 403: 501-502.

7 Beaujean N, Hartshorne G, Cavilla J et al: Non-conservation of mammalian preimplantation methylation dynamics. Curr Biol 2004; 14: R266-R267.
8 Santos F, Dean W: Epigenetic reprogramming during early development in mammals. Reproduction 2004; 127: 643-651.

9 Santos F, Hendrich B, Reik W, Dean W: Dynamic reprogramming of DNA methylation in the early mouse embryo. Dev Biol 2002; 241: 172-182.

10 Howlett SK, Reik W: Methylation levels of maternal and paternal genomes during preimplantation development. Development 1991; 113: 119-127.

11 Omizawa S, Kobayashi H, Watanabe $\mathrm{T}$ et al: Dynamic stage-specific changes in imprinted differentially methylated regions during early mammalian development and prevalence of non-CpG methylation in oocytes. Development 2011; 138: 811-820.

12 Zhao MT, Rivera RM, Prather RS: Locus-specific DNA methylation reprogramming during early porcine embryogenesis. Biol Reprod 2013; 88: 48.

13 Paranjpe SS, Veenstra GJ: Establishing pluripotency in early development. Biochim Biophys Acta 2015; 1849: 626-636.

14 Magdinier F, D'estaing SG, Peinado $C$ et al: Epigenetic marks at BRCA1 and p53 coding sequences in early human embryogenesis. Mol Hum Reprod 2002; 8: 630-635.

15 Muttukrishna S, Mcgarrigle H, Wakim R, Khadum I, Ranieri DM, Serhal P: Antral follicle count, anti-mullerian hormone and inhibin $\mathrm{B}$ : predictors of ovarian response in assisted reproductive technology? BJOG 2005; 112: 1384-1390.

16 Sahu B, Ozturk O, Deo N, Fordham K, Ranierri M, Serhal P: Response to controlled ovarian stimulation and oocyte quality in women with myotonic dystrophy type I. J Assist Reprod Genet 2008; 25: 1-5.

17 Bolton VN, Hawes SM, Taylor CT, Parsons JH: Development of spare human preimplantation embryos in vitro: an analysis of the correlations among gross morphology, cleavage rates, and development to the blastocyst. J In Vitro Fert Embryo Transf 1989; 6: 30-35.

18 Esteller M, Silva JM, Dominguez G et al: Promoter hypermethylation and BRCA1 inactivation in sporadic breast and ovarian tumors. J Natl Cancer Inst 2000; 92: 564-569.

19 Ibala-Romdhane S, Al-Khtib M, Khoueiry R, Blachere T, Guerin JF, Lefevre A: Analysis of $\mathrm{H} 19$ methylation in control and abnormal human embryos, sperm and oocytes. Eur $\mathrm{J}$ Hum Genet 2011; 19: 1138-1143.

20 Monk M, Salpekar A: Expression of imprinted genes in human preimplantation development. Mol Cell Endocrinol 2001; 183(Suppl 1): S35-S40.

21 Khoueiry R, Ibala-Romdhane S, AI-Khtib M: Abnormal methylation of KCNQ10T1 and differential methylation of $\mathrm{H} 19$ imprinting control regions in human ICSI embryos. Zygote 2012; 21: 129-138.

22 Huntriss J, Daniels R, Bolton V, Monk M: Imprinted expression of SNRPN in human preimplantation embryos. Am J Hum Genet 1998; 63: 1009-1014.

23 Lighten AD, Hardy K, Winston RM, Moore GE: IGF2 is parentally imprinted in human preimplantation embryos. Nat Genet 1997; 15: 122-123.

24 DeBaun MR, Niemitz EL, Feinberg AP: Association of in vitro fertilization with Beckwith-Wiedemann syndrome and epigenetic alterations of LIT1 and H19. Am J Hum Genet 2003; 72: 156-160.

25 Orstavik KH, Eiklid K, Van Der Hagen CB: Another case of imprinting defect in a girl with Angelman syndrome who was conceived by intracytoplasmic semen injection. Am J Hum Genet 2003; 72: 218-219.

26 Gicquel C, Gaston V, Mandelbaum J, Siffroi JP. Flahault A, Le Bouc Y: In vitro fertilization may increase the risk of Beckwith-Wiedemann syndrome related to the abnormal imprinting of the KCN1OT gene. Am J Hum Genet 2003; 72: 1338-1341.

27 Sato A, Otsu E, Negishi H, Utsunomiya T, Arima T: Aberrant DNA methylation of imprinted loci in superovulated oocytes. Hum Reprod 2007; 22: 26-35.

28 Orban TI, Olah E: Emerging roles of BRCA1 alternative splicing. Mol Pathol 2003; 56: 191-197.

29 Lixia M, Zhijian C, Chao S, Chaojiang G, Congyi Z: Alternative splicing of breast cancer associated gene BRCA1 from breast cancer cell line. J Biochem Mol Biol 2007; 40: 15-21.

30 Wilson CA, Payton MN, Elliott GS et al: Differential subcellular localization, expression and biological toxicity of BRCA1 and the splice variant BRCA1-delta11b. Oncogene 1997; 14: 1-16.

31 Tammaro C, Raponi M, Wilson DI, Baralle D: BRCA1 exon 11 alternative splicing, multiple functions and the association with cancer. Biochem Soc Trans 2012; 40: 768-772.

32 Borel C, Ferreira PG, Santoni $\mathrm{F}$ et al: Biased allelic expression in human primary fibroblast single cells. Am J Hum Genet 2015; 96: 70-80.

33 Senst N, Llacuachaqui M, Lubinski J et al: Parental origin of mutation and the risk of breast cancer in a prospective study of women with a BRCA1 or BRCA2 mutation. Clin Genet 2012; 84: 43-46.

34 Ellberg C, Jernström H, Broberg P, Borg $\AA$, Olsson $\mathrm{H}$ : Impact of a paternal origin of germline BRCA1/2 mutations on the age at breast and ovarian cancer diagnosis in a Southern Swedish cohort. Genes Chromosomes Cancer 2014; 54: 39-50.

Supplementary Information accompanies this paper on European Journal of Human Genetics website (http://www.nature.com/ejhg) 